\title{
Branches of the Anterior Cerebral Artery Near the Anterior Communicating Artery Complex: an Anatomic Study and Surgical Perspective
}

\author{
Emel Avci, Damirez Fossett, Mehmet Aslan*, Ayhan ATTAR*, \\ and Nihat EGEMEN*
}

\begin{abstract}
Department of Neurosurgery, The Harvey Ammerman Neurosurgical Laboratory, George Washington University, Washington, D.C., U.S.A; * Department of Neurosurgery, Ankara University School of Medicine, Ankara, Turkey
\end{abstract}

\begin{abstract}
The anatomy of the branches of the anterior cerebral artery (ACA) near the anterior communicating artery (ACoA) complex were investigated to minimize neurovascular morbidity caused by surgical procedures performed in this region. Thirty-one cadaver brains were perfused with colored silicone, fixed, and studied under the operating microscope. The recurrent artery of Heubner (RAH), orbitofrontal artery (OFA), and frontopolar artery (FPA) were identified as the branches of the ACA arising near the ACoA complex. The OFA and FPA were identified in all hemispheres. Forty-nine (64\%) of a total of 77 RAHs arose from the $A_{2}$ segment. The OFA always arose from the $A_{2}$ segment, was consistently the smallest branch, and coursed to the gyrus rectus, olfactory tract, and olfactory bulb. The mean distance between the ACoA and the OFA was $5.96 \mathrm{~mm}$. The FPA arose from the $A_{2}$ segment in $95 \%$ of the specimens, and coursed to the medial subfrontal region. The mean distance between the ACoA and the FPA was $14.6 \mathrm{~mm}$. The RAH, OFA, and the FPA are three branches that arise from the ACA near the ACoA complex. These vessels have similar diameters, but can be distinguished by the final destination. Distinguishing these vessels is important since the consequences of injury or occlusion of the FPA and OFA are significantly less than of the RAH.
\end{abstract}

Key words: recurrent artery of Heubner, orbitofrontal artery, frontopolar artery, anterior cerebral artery

\section{Introduction}

Many studies have investigated the anatomy of the anterior cerebral-anterior communicating artery (ACA-ACoA) vascular complex. ${ }^{1-10}$ ) The vascular relationships of this region are especially important as pathology requiring surgical treatment is often found here. Successful surgery without incurring significant neurovascular morbidity in this region depends on knowledge of its detailed vascular anatomy. ${ }^{8)}$ We report our study of the anatomy of the branches of the ACA near the ACoA junction in 31 cadavers, with specific attention given to the recurrent artery of Heubner (RAH), the orbitofrontal artery (OFA), and the frontopolar artery (FPA).

\section{Materials and Methods}

Thirty-one human brains were obtained at routine autopsies performed 4-8 hours postmortem. The internal carotid arteries and the basilar artery were cannulated, irrigated with saline to remove luminal clots, and then perfused with red-colored silicone. The brains were then placed in a $10 \%$ formaldehyde solution for one week. Microdissection was performed under an operating microscope (Carl Zeiss Universal S2; Carl Zeiss, Jena, Germany). Measurements were made with 8 inch $/ 200 \mathrm{~mm}$ electronic digital calipers (Marathon, Richmand Hill, Ontario, Canada).

Received July 24, 2001; Accepted February 18, 2003

Author's present address: Emel Avcı, M.D., Sosyal Sigortalar Bölge Hastanesi, Gaziantep, Turkey. 


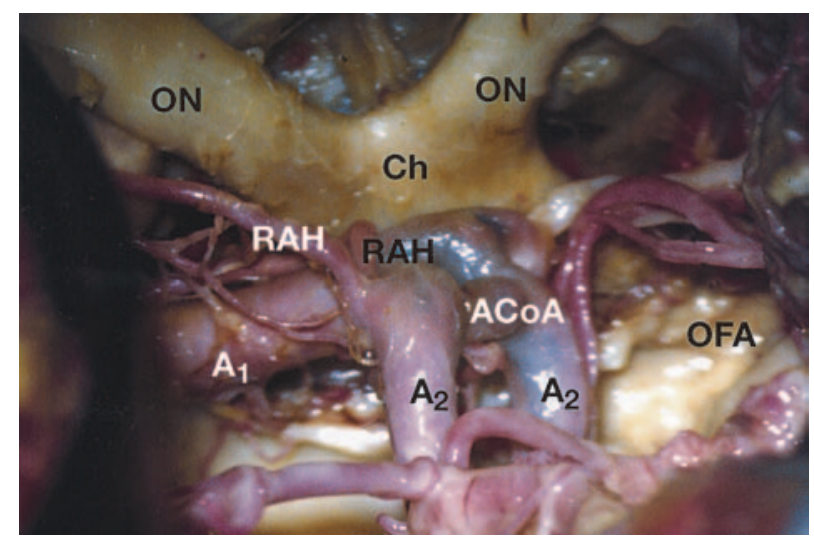

Fig. 1 Photograph illustrating a fenestrated anterior communicating artery (ACoA) complex with two recurrent arteries of Heubner (RAHs) originating at the level of the ACoA junction. The optic chiasm (Ch) is well visualized. $A_{1}$ and $A_{2}$ : segments of the anterior cerebral artery, OF $A$ : orbitofrontal artery, ON: optic nerve.

\section{Results}

The vascular anatomy of the ACA-ACoA complex was examined in 62 cerebral hemispheres. The diameter of the ACA proximal to the ACoA ranged from 1.28-3.29 mm (mean $2.1 \mathrm{~mm}$ ) and that distal to the ACoA ranged from 1.24-2.98 mm (mean $2.0 \mathrm{~mm})$. The RAH was absent in only one $(1.6 \%)$ hemisphere. A single RAH was present in 46 (74.2\%) hemispheres, two RAHs were found in 14 (22.6\%) hemispheres (Fig. 1), and three RAHs in one (1.6\%) hemisphere. The mean diameter was $0.45 \mathrm{~mm}$ and the mean length was $17.4 \mathrm{~mm}$.

Forty-nine (64\%) of the 77 RAHs arose from the $A_{2}$ segment, $22(29 \%)$ from the ACoA-ACA junction, and six (8\%) from the $A_{1}$ segment. The number of perforating branches from RAH varied from three to 11 (mean 4). Perforators could be separated by region of penetration. An olfactory branch was seen in eight $(13 \%)$ of the hemispheres, a frontal branch in two (3\%), and a sylvian fissure branch in $12(19 \%)$. Perforators went to the center of the anterior perforating substance (APS) in 17 (27\%), to the medial part in $11(18 \%)$, and to the lateral part in $12(19 \%)$ (Fig. 2). The RAH was directed superiorly in 29 cases (38\%) (Fig. 3), anteriorly in 40 (52\%) (Fig. 4), and posteriorly in eight (10\%).

The OFA and FPA were identified in all our specimens (Figs. 5 and 6). The OFA diameter ranged from 0.21-1.85 mm (mean 0.79 mm). The OFA was consistently the smallest branch of the whole distal

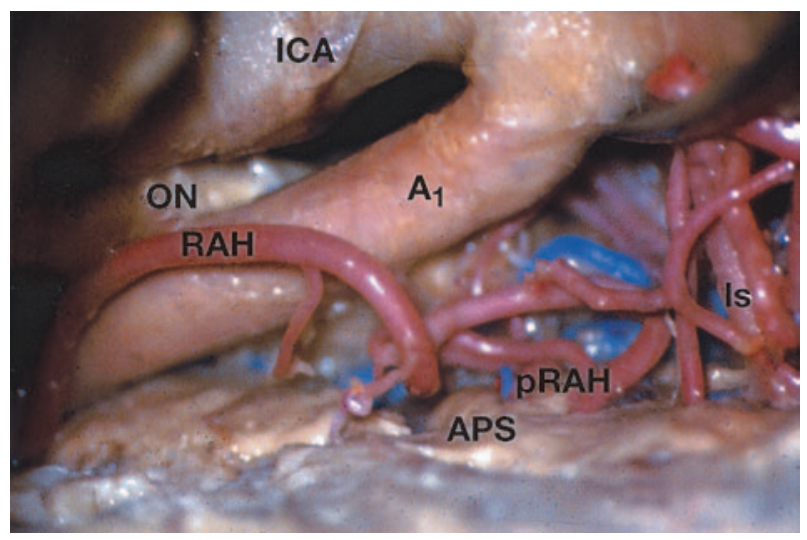

Fig. 2 Photograph showing the recurrent artery of Heubner (RAH) coursing to the anterior perforating substance (APS). The lenticulostriate branches (ls) of the middle cerebral artery (MCA) are also seen. Note that the size of the RAH perforators (pRAH) is similar to that of the lenticulostriate branches of the MCA. $A_{1}$ : segment of the anterior cerebral artery, ICA: internal carotid artery, ON: optic nerve.

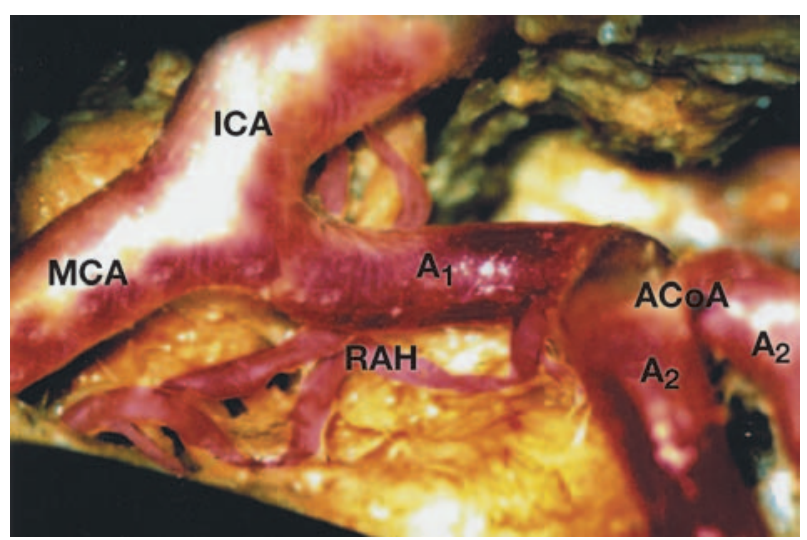

Fig. 3 Photograph showing the anterior cerebral circulating complex with the recurrent artery of Heubner (RAH) arising from the distal $A_{1}$. Note the superior course of the RAH. $A_{1}$ and $A_{2}$ : segments of the anterior cerebral artery, ACoA: anterior communicating artery, ICA: internal carotid artery, MCA: middle cerebral artery.

ACA, and always arose from the $A_{2}$ segment of the ACA as a single trunk and ran to the gyrus rectus, olfactory tract, and olfactory bulb. The distance between the ACoA and the OFA ranged from 2.61$8.22 \mathrm{~mm}$ (mean $5.96 \mathrm{~mm}$ ). The FPA arose from the $A_{2}$ segment in $95 \%$ of our specimens. The other $5 \%$ of FPAs arose from the $A_{3}$ segment of the ACA. The 


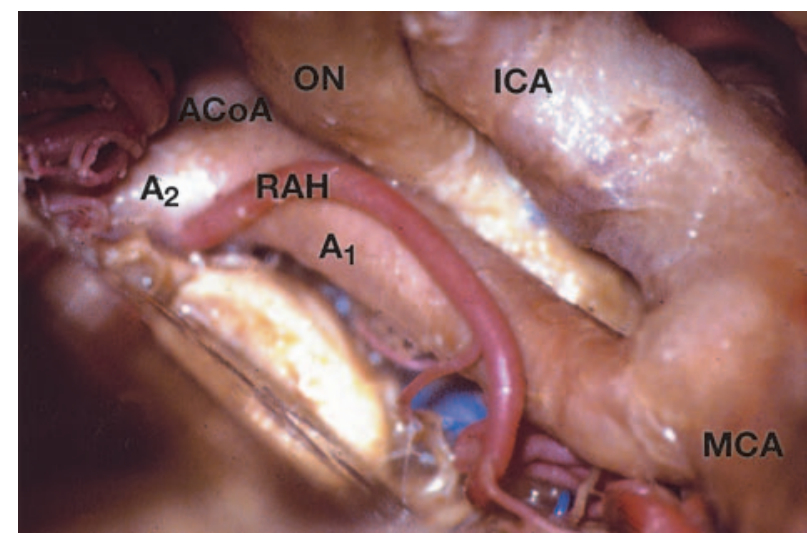

Fig. 4 Photograph illustrating an anterior course of the recurrent artery of Heubner (RAH). $A_{1}$ and $A_{2}$ : segments of the anterior cerebral artery, ACoA: anterior communicating artery, ICA: internal carotid artery, MCA: middle cerebral artery, $\mathrm{ON}$ : optic nerve.

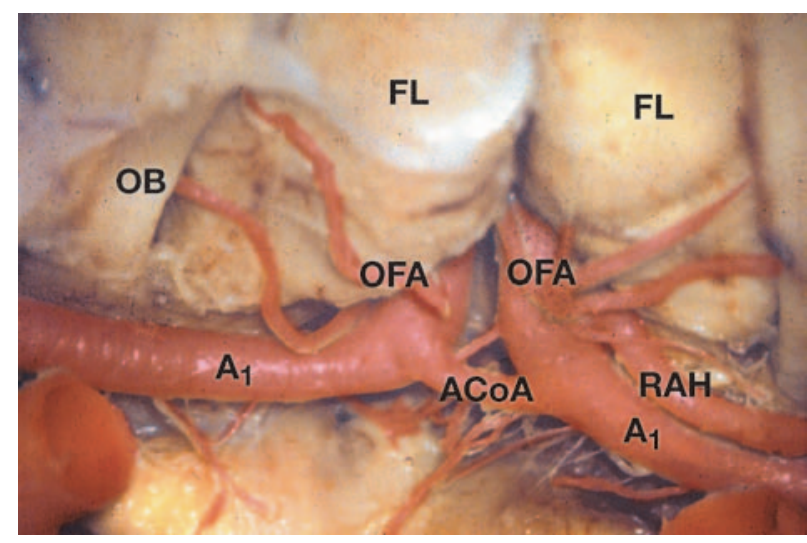

Fig. 5 Photograph showing the recurrent artery of Heubner (RAH) and orbitofrontal arteries (OFAs). The destination and course of the OFA can be distinguished from those of the RAH. $A_{1}$ : segment of the anterior cerebral artery, ACoA: anterior communicating artery, FL: frontal lobe, OB: olfactory bulb.

diameter ranged from $0.39-1.22 \mathrm{~mm}$ (mean 1.27 $\mathrm{mm}$ ), and ran to the medial subfrontal aspect of the anterior frontal lobe. The distance between the ACoA and the FPA ranged from $5.63-18.16 \mathrm{~mm}$ (mean $14.6 \mathrm{~mm}$ ).

\section{Discussion}

Table 1 shows a comparative review of data from the literature ${ }^{1,3,6,8)}$ and our own study of the RAH. The overall preponderance of data suggests that the

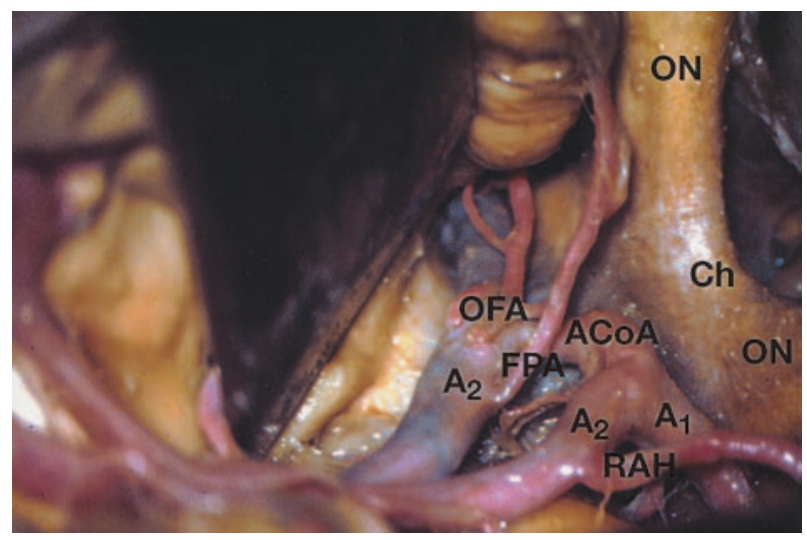

Fig. 6 Photograph showing an ipsilateral recurrent artery of Heubner (RAH) arising from the $\mathrm{A}_{2}$ segment. The $\mathrm{RAH}$, orbitofrontal artery (OFA), and frontopolar artery (FPA) arise in the anterior cerebral artery (ACA)anterior communicating artery (ACoA) region on the contralateral side. Note the proximity in size and point of origin of these three branches of the ACA. $A_{1}$ and $A_{2}$ : segments of the anterior cerebral artery, $\mathrm{Ch}$ : optic chiasm, $\mathrm{ON}$ : optic nerve.

RAH most typically arises as a single artery, but our data support the notion of variability in the number of RAHs in any one hemisphere. The take-off of the RAH may be at the same level in both cerebral hemispheres, ${ }^{12)}$ directly opposite the ACoA or in very close proximity in $90 \%$ of cases, ${ }^{2)}$ or the artery may arise either opposite to or within $5 \mathrm{~mm}$ of the ACoA in $95 \%$ of cases. ${ }^{5}$ ) Our study shows that the RAH appears to arise most often in close proximity to the ACoA junction.

Three courses of the RAH have been described. In type I, or the superior course, RAH follows the superior wall of the $A_{1}$ segment. In type II, or the anterior course, the artery maintains an anterior position in relation to the $A_{1}$ segment. In type III, or the posterior course, the artery follows a posterior course into the APS. ${ }^{3)}$ The courses of the RAH are comparable between the reviewed series and our own study. ${ }^{1,3,5,6)}$ Our study also demonstrated a significant number of perforating branches from the RAH. In concordance with previous studies, our study found the point of penetration of $83 \%$ of the RAH perforators was the APS and the sylvian fissure. An average of four perforators arose from the $\mathrm{RAH}$ in the region of the carotid bifurcation. These perforators had a diameter similar to that of the lenticulostriate branches of the middle cerebral artery and preservation of these vessels is as important as preservation of the lenticulostriate 
Table 1 Findings of various studies of the recurrent artery of Heubner (RAH)

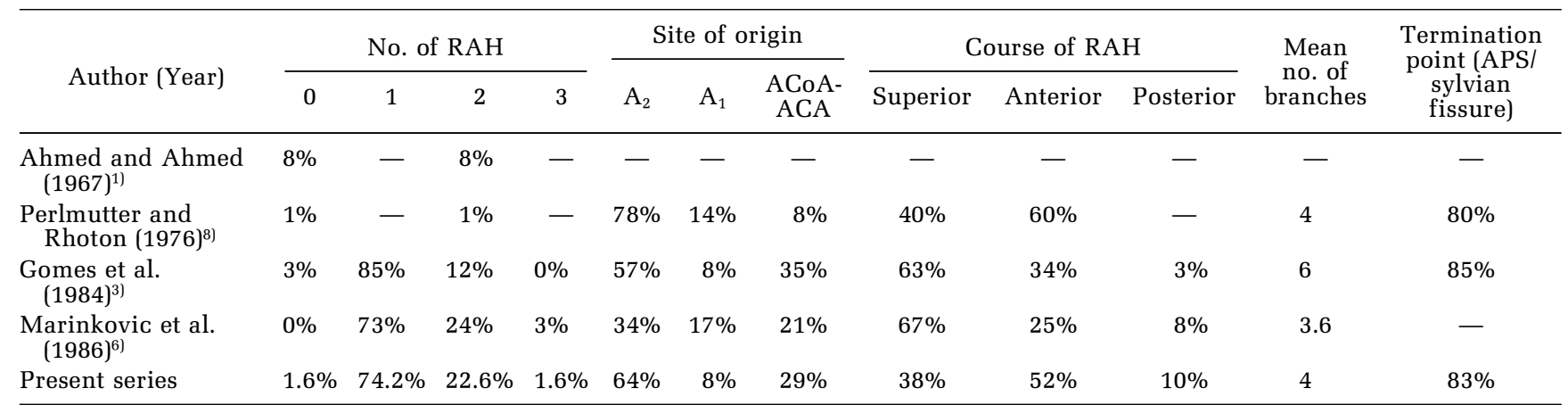

$A_{1}$ and $A_{2}$ : segments of the anterior cerebral artery, ACA: anterior cerebral artery, ACoA: anterior communicating artery, APS: anterior perforating substance.

branches.

The OFA is not always the first cortical branch of the proximal $A_{2}$ segment of the ACA. In our study, the RAH arose either at the ACoA-ACA junction or along the proximal portion of the $A_{2}$ segment in $92 \%$ of cases. The OFA can be distinguished from the RAH because it generally courses downward and forward to reach the region of the gyrus rectus, olfactory tract, and olfactory bulb. The FPA arose on average $9 \mathrm{~mm}$ distal to the OFA and coursed anteriorly toward the medial subfrontal surface. The FPA arises more distally along the $\mathrm{A}_{2}$ segment, and the course is directed anteriorly instead of laterally, so can be distinguished from the RAH. Small FPA and OFA may sometimes have to be sacrificed for clipping of ACoA aneurysms, but the RAH should never intentionally be sacrificed. ${ }^{10)}$

The RAH is important in the surgery of ACoA aneurysms or internal carotid artery bifurcation aneurysms, and for the resection of parasellar neoplasms. The RAH, OFA, and FPA all arise near the ACoA-ACA junction, and the diameter of each is similar, so correct identification of the RAH may be difficult. An ACoA aneurysm that projects anteriorly or superiorly may hide the contralateral $A_{1}$ segment. With marked inequality of the $A_{1}$ segments, the smaller artery may be mistaken for an RAH or an OFA. The $A_{2}$ segments are regularly attached to the aneurysm fundus, especially if posteriorly projecting. The lateral course of the RAH results in attachment to an aneurysm fundus which projects anterolaterally or inferolaterally. ${ }^{11)}$ The most important differentiating characteristics between these three vessels are the direction of the course and the final destination. Knowledge of the pertinent vascular anatomy can distinguish these arteries during surgical procedures in the region of the ACA-ACoA complex, which is immensely important since the conse- quences of injury or occlusion of the FPA and OFA are significantly less than of the RAH.

\section{References}

1) Ahmed DS, Ahmed RH: The recurrent branch of the anterior cerebral artery. Anat Rec 157: 699-700, 1967

2) Dunker RO, Harris AB: Surgical anatomy of the proximal anterior cerebral artery. J Neurosurg 44: 359-367, 1976

3) Gomes F, Dujovny M, Umansky F, Ausman JI, Diaz FG, Ray WJ, Mirchandani HG: Microsurgical anatomy of the recurrent artery of Heubner. J Neurosurg 60: 130-139, 1984

4) Kaplan HA: The lateral perforating branches of anterior and middle cerebral arteries. J Neurosurg 23: 305-310, 1965

5) Kribs M, Kleihues $P$ : The recurrent artery of Heubner. A morphological study of the blood supply of the rostral basal ganglia in normal and pathological conditions, in Zulch KJ (ed): Cerebral Circulation and Stroke. New York, Springer-Verlag, 1971, pp 40-56

6) Marinkovic S, Milisavljevic M, Kovacevi M: Anatomical bases for surgical approach to the initial segment of the anterior cerebral artery. Surg Radiol Anat 8: $7-18,1986$

7) Moscow N, Michotey P, Salamon G: Anatomy of the cortical branches of the anterior cerebral artery, in Newton TH, Potts DG (eds): Radiology of the Skull and Brain, vol 3. St Louis, CV Mosby, 1974, pp 1411-1420

8) Perlmutter D, Rhoton AL Jr: Microsurgical anatomy of the anterior cerebral-anterior communicatingrecurrent artery complex. J Neurosurg 45: 259-272, 1976

9) Perlmutter D, Rhoton AL Jr: Microsurgical anatomy of the distal anterior cerebral artery. J Neurosurg 49: 204-228, 1978

10) Solomon RA: Anterior communicating artery aneurysms. Neurosurgery 48: 119-123, 2001

11) Yasargil MG: Microneurosurgery, vol II. New York, 
Georg Thieme Verlag, 1984, pp 178-179

12) Yasargil MG, Smith RD: Management of aneurysms of anterior circulation by intracranial procedures, in Youmans JR (ed): Neurological Surgery, ed 2, vol 3. Philadelphia, WB Saunders, 1982, pp 1663-1696

Address reprint requests to: E. Avc1, M.D., Sosyal Sigortalar Bölge Hastanesi, Fevzi Çakmak Bulvarı, 27060, Beyin Cerrahisi Kliniği, Kat: 2 Gaziantep, Turkey.

e-mail: avciemel@hotmail.com

\section{Commentary}

Dr. Avci and colleagues have reported an elegant cadaveric study of the microvascular anatomy near the anterior communicating complex. In particular, the authors have outlined the course of the recurrent artery of Heubner, the fronto-polar artery and the orbito-frontal artery. As demonstrated in previous anatomic studies, variability in the anatomy of the anterior communicating complex is the rule rather than the exception. Appreciation of that variability is essential to the neurosurgeon operating on lesions in this region. As emphasized by the authors, extraordinary care must be taken to preserve the recurrent artery of Heubner, as injury to this small but important structure can result in devastating neurological consequences. The authors are to be congratulated for their beautiful cadaveric dissections and illustrations.

Daniel L. BARROW, M.D. Department of Neurosurgery

The Emory Clinic Atlanta, Georgia, U.S.A.

The authors have performed detailed and delicate dissections of arterial branches in the anterior communicating region in a large number of brains, and shown considerable though not surprising variations. Important details of the vascular anatomy are well demonstrated, in particular that of the important recurrent artery, damage to which can cause a devastating stroke. The variability shown in the origin, course and destination of this artery was shown, along with the important point that it was absent in only one of 62 hemispheres, and was duplicated in nearly a quarter of cases.

These points emphasize the vital importance, during surgery, of defining the anatomy of these branches exactly, to avoid damaging them or including them in a clip. Useful tips about the different directions of the recurrent artery and other branches are given. Although the others are less important they should still be preserved where possible, especially avoiding damage to both orbito-frontal arteries. As noted, the recurrent artery must also be identified and preserved during surgery for carotid bifurcation aneurysms.

Nicholas W. C. DorsCh, M.D., F.R.C.S., F.R.A.C.S. Department of Neurosurgery Westmead Hospital Sydney, Australia

The authors performed an anatomic study using cadaver brains to provide data relevant to the surgical treatment of anterior communicating aneurysms, giving special reference to the recurrent artery of Heubner, an orbitofrontal artery, and the frontopolar artery of the anterior cerebral artery. As an occlusion of Heubner's artery may cause hemiparesis and aphasia, they emphasized the importance of preserving the artery during surgery for an anterior communicating aneurysm. In addition to their study, I would like to stress that the anterior communicating artery is the origin of as many as four perforating branches to the dorsal surface of the optic chiasm and the suprachiasmatic area. These perforating branches perfuse the fornix, corpus callosum, and septal region. Their occlusion can result in personality and memory disturbances.

Kyu Chang LEE, M.D., F.A.H.A. Department of Neurosurgery Yonsei University College of Medicine Seoul, R.O.K. 